\title{
Chronic Graft versus Host Disease but not the Intensity of Conditioning Has Impact on Survival after Allogeneic Hematopoietic Stem Cell Transplantation for Advanced Hematological Diseases
}

\author{
Inken Hilgendorf ${ }^{a}$ Daniel Wolff ${ }^{b}$ Axel Nogai ${ }^{c}$ Günther Kundt ${ }^{d}$ Joachim Hahn $^{b}$ Ernst Holler $^{b}$ \\ Lutz Uharek $^{c}$ Christian Junghanss $^{\mathrm{a}}$ Malte Leithäuser ${ }^{\mathrm{a}}$ Eckhard Thiel $^{\mathrm{c}}$ Mathias Freund $^{\mathrm{a}}$ Christoph Kahl $^{\mathrm{a}}$ \\ aDepartment of Hematology, Oncology, Palliative Care, University of Rostock, \\ ${ }^{b}$ Department of Hematology and Oncology, University of Regensburg, 'Medizinische Klinik III, Campus Benjamin Franklin, Charité Berlin, \\ ${ }^{\mathrm{d} D e p a r t m e n t}$ of Medical Informatics and Biometry, University of Rostock, Germany
}

\section{Keywords}

Hematological malignancies, advanced · Allogeneic stem cell transplantation . Graft versus host disease, chronic . Myeloablative conditioning $\cdot$ Reduced intensity conditioning

\section{Summary}

Background: Allogeneic hematopoietic stem cell transplantation (alloHSCT) is often performed in cases of advanced hematological diseases, but because of the associated mortality and a high risk of relapse it is life prolonging only in some patients. Patients and Methods: A retrospective multi-center analysis of 401 patients was conducted to analyze the variables associated with outcome after alloHSCT in advanced hematological diseases. The Cox proportional hazards model was used to assess the independence of overall survival (OS) and disease-free survival (DFS) from prognostic factors in a multivariate model. Results: The 5-year OS and DFS were 27.3 and $21.1 \%$ respectively. Multivariate analysis showed that the underlying malignancy had a significant influence on OS and DFS ( $p<0.001$ and $p<0.011$, respectively), whereas development of severe acute graft versus host disease (GvHD) had a negative impact on OS $(p<0.001)$. Development of chronic GvHD showed a trend to a better OS $(p=0.085)$ and DFS $(p=0.199)$. No impact was seen for the intensity of conditioning. Conclusion: Development of chronic GvHD but not the conditioning regimen improved the outcome after alloHSCT for advanced malignancies, underlining the importance of immunological rather than cytotoxic effects.

\section{Schlüsselwörter}

Hämatologische Erkrankung, fortgeschrittene .

Allogene Stammzelltransplantation · Graft-versus-HostReaktion, chronische $\cdot$ Konditionierung: myeloablative, intensitätsreduzierte

\section{Zusammenfassung}

Hintergrund: Bei fortgeschrittenen malignen hämatologischen Erkrankungen wird häufig eine allogene Stammzelltransplantation (alloHSZT) durchgeführt, die jedoch auf Grund der hohen behandlungsassoziierten Mortalität und einem hohen Rezidivrisiko nur bei einem Teil der Patienten lebensverlängernd ist. Patienten und Methoden: Um Variablen zu analysieren, die mit dem Erfolg einer alloHSZT bei fortgeschrittenen malignen hämatologischen Erkrankungen assoziiert sind, wurde eine retrospektive, multizentrische Erhebung an 401 Patienten durchgeführt. Dabei wurden unabhängige prognostische Faktoren bezüglich des Gesamtüberlebens (OS) und des erkrankungsfreien Überlebens (DFS) in der multivariaten Analyse unter Verwendung des proportionalen HazardModells (Cox-Modell) untersucht. Ergebnisse: Das OS und DFS betrug nach 5 Jahren 27,3 bzw. 21,1\%. In der multivariaten Analyse konnte gezeigt werden, dass die zugrunde liegende maligne Erkrankung einen signifikanten Einfluss auf das OS und das DFS hat ( $p<0,001$ und 0,011), während das Auftreten einer schweren akuten Graft-versus-Host-Reaktion (GvHD) einen negativen Einfluss auf das OS hatte $(p<0,001)$. Das Auftreten einer chronischen GvHD zeigte einen Trend für ein besseres OS $(p=0,085)$ und DFS $(p=0,199)$. Die Intensität der Konditionierungstherapie hatte hingegen keinen Einfluss auf das OS und DFS. Schlussfolgerung: Die Verbesserung des Ergebnisses nach alloHSZT bei fortgeschrittenen hämatologischen Erkrankungen infolge des Auftretens einer chronischen GvHD aber nicht infolge der Konditionierungstherapie unterstreicht die Bedeutung des mit der chronischen GvHD assoziierten Graft-versus-Leukämie-Effekts.

Inken Hilgendorf, MD

Department of Hematology, Oncology, Palliative Care University of Rostock

Ernst-Heydemann-Str.6, 18055 Rostock, Germany

Tel. +49 381 494-7420, Fax -7422

inken.hilgendorf@med.uni-rostock.de 


\section{Introduction}

Treatment of malignant hematological diseases has been dramatically improved by the introduction of allogeneic hematopoietic stem cell transplantation (alloHSCT) [1]. Factors influencing the outcome after alloHSCT include tumor burden, donor selection, disease stage, the development of graft versus host disease (GvHD) and the graft composition [2-7]. Despite the fact that the outcome after alloHSCT in relapsed or refractory hematological diseases is poor, it is often performed since it is the only remaining curative option. However, this procedure is debatable. Due to its high morbidity and mortality, it potentially shortens the remaining life time of patients without any clinical benefit. Therefore, decisions regarding the appropriateness of alloHSCT for a given patient should involve careful consideration of the risks associated with alloHSCT and the likelihood of cure. The intention of this retrospective analysis was to investigate, by identifying parameters with prognostic influence, which patients suffering from advanced hematological disease could benefit from an alloHSCT and should therefore be offered this procedure.

\section{Patients, Material and Methods}

\section{Eligibility Criteria}

Between 1993 and 2009, 401 patients with advanced hematological diseases were treated with different transplantation protocols within the Division of Hematology and Oncology, University of Rostock, the Medical Clinic III, Campus Benjamin Franklin, Charite Berlin and the Department of Hematology and Oncology, University of Regensburg. The Institution Review Boards (IRB) at each of the collaborating centers approved the protocols. Patients signed consent forms that were in accordance with the Declaration of Helsinki and approved by the local IRBs

Patients, Conditioning Regimen and Postgrafting Immunosuppression An advanced stage of a disease was defined as refractory disease (RD), partial remission (PR) or more than 2 complete remissions (CR) at time of alloHSCT [8]. A majority of the 401 patients had acute myeloid leukemia $(\mathrm{AML}, \mathrm{n}=188)$ or non-Hodgkin's lymphoma (NHL, $\mathrm{n}=80$ ), followed by patients with myeloproliferative syndromes (MPS, $\mathrm{n}=54$ ), acute lymphoblastic leukemia (ALL, $\mathrm{n}=47$ ), multiple myeloma (MM, $\mathrm{n}=24)$ or Hodgkin's disease (HD, $\mathrm{n}=8$ ). The median age of patients was 46.3 years (range 13-70) with 254 male and 147 female patients. Patients received either related $(\mathrm{n}=172)$ or unrelated $(\mathrm{n}=229)$ HLA (human leukocyte antigen)-identical grafts. Patients and donors were matched for HLA-A, B, and C at least at the antigen level and for DRB1 and DQB1 at the allele level. 356 patients received peripheral blood stem cell grafts and 45 received bone marrow. In $333(83.0 \%)$ of the 401 patients the remission status at time of alloHSCT was known. Pre-transplantion, only 33 patients $(9.9 \%)$ were in CR for more than the second time, whereas most of the patients had measurable disease at time of transplan-

Table 1. Patient characteristics

\begin{tabular}{|c|c|c|c|c|c|c|}
\hline & AML & NHL & MPS & ALL & $\mathrm{MM}$ & Hodgkin \\
\hline \multicolumn{7}{|l|}{ Patient age, years } \\
\hline Median & 47 & 44 & 50 & 36 & 53 & 39 \\
\hline \multirow[t]{2}{*}{ Range } & $13-69$ & $15-70$ & $17-65$ & $15-58$ & $41-65$ & $18-54$ \\
\hline & $\mathrm{n}$ & $\mathrm{n}$ & $\mathrm{n}$ & $\mathrm{n}$ & $\mathrm{n}$ & $\mathrm{n}$ \\
\hline Total & 188 & 80 & 54 & 47 & 24 & 8 \\
\hline \multicolumn{7}{|l|}{ Patient gender } \\
\hline Male & 115 & 53 & 41 & 29 & 12 & 4 \\
\hline Female & 73 & 27 & 13 & 18 & 12 & 4 \\
\hline \multicolumn{7}{|l|}{ Source } \\
\hline PBSC & 171 & 69 & 50 & 45 & 20 & 1 \\
\hline Bone marrow & 17 & 11 & 4 & 2 & 4 & 7 \\
\hline \multicolumn{7}{|l|}{ Donor } \\
\hline Related & 75 & 48 & 10 & 24 & 11 & 4 \\
\hline Unrelated & 113 & 32 & 44 & 23 & 13 & 4 \\
\hline \multicolumn{7}{|l|}{ Status before HSCT } \\
\hline 1 complete or partial remission & 19 & 10 & 0 & 8 & 6 & 0 \\
\hline Stable disease & 4 & 3 & 0 & 0 & 0 & 0 \\
\hline Refractory disease & 39 & 17 & 0 & 10 & 5 & 2 \\
\hline Relapse & 42 & 14 & 0 & 23 & 2 & 2 \\
\hline Unknown & 2 & 8 & 54 & 0 & 4 & 0 \\
\hline \multicolumn{7}{|l|}{ Conditioning } \\
\hline Busulfan-based & 12 & 0 & 16 & 0 & 0 & 0 \\
\hline FBM & 28 & 23 & 5 & 3 & 10 & 2 \\
\hline FLAMSA & 36 & 0 & 0 & 1 & 0 & 0 \\
\hline MitoFlag & 6 & 0 & 0 & 0 & 0 & 0 \\
\hline Treosulfan-based & 31 & 11 & 11 & 4 & 9 & 1 \\
\hline HD-TBI & 45 & 33 & 18 & 24 & 5 & 4 \\
\hline TBI 2Gy & 10 & 0 & 3 & 0 & 0 & 0 \\
\hline Others & 20 & 13 & 1 & 15 & 0 & 1 \\
\hline
\end{tabular}

$\mathrm{AML}=$ acute myeloid leukemia, $\mathrm{NHL}=$ non-Hodgkin's lymphoma, $\mathrm{MPS}=$ myeloproliferative syndrome, $\mathrm{ALL}=$ acute lymphoblastic leukemia, $\mathrm{MM}=$ multiple myeloma, PBSC $=$ peripheral blood stem cells, HSCT = hematopoietic stem cell transplantation, FBM = fludarabine, BCNU, melphalan, FLAMSA = fludarabine, amsacrine, HD-TBI = high-dose total body irradiation. 
Fig 1. Overall survival (OS) (A) and diseasefree survival (DFS) (B) in 401 patients undergoing hematopoietic stem cell transplantation (HSCT)
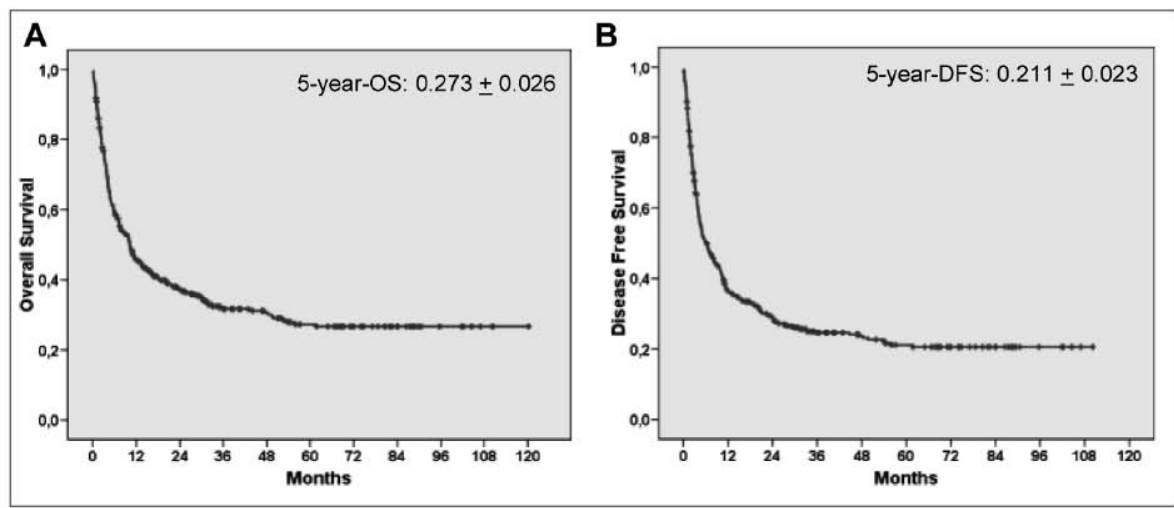

tation; these patients included 137 patients $(41.1 \%)$ in PR, 7 (2.1\%) in stable disease (SD) and $156(46.8 \%)$ with RD. Transplantation was performed in 33 patients ( $70 \%$ ) with ALL with active relapsed disease, in 8 patients $(17 \%)$ with PR and in 6 patients $(12 \%)$ with CR. None of the patients with $\mathrm{HD}$ was in $\mathrm{CR}$ at time of transplant, 4 patients (50\%) were in PR, and $4(50 \%)$ suffered from active relapsed disease. 47 patients had a Karnofsky index of 100,125 an index of 90,109 an index of 80 and 65 an index of 70. Patients' characteristics are shown in table 1.

The patients received various conditioning regimens and immunosuppression protocols. The majority were treated with a reduced-intensity conditioning (RIC, $\mathrm{n}=334$ ) protocol, 54 patients received myeloablative conditioning (MAC) and 13 patients were transplanted after non-myeloablative conditioning. GvHD was evaluated according to the Keystone criteria (acute (a) GvHD) and the classification according to Shulman et al. (chronic (c) GVHD) $[9,10]$.

\section{Statistical Analysis}

All data were stored and analyzed using the SPSS statistical package 17.0 (SPSS Inc. Chicago, IL, USA). Statistical analysis was realized in agreement with Statistical Guidelines of EBMT (European Group for Blood and Marrow Transplantation). The statistics computed included mean and standard deviations of continuous variables, frequencies and relative frequencies of categorical factors. The Cox proportional hazards model was used to assess the independence of overall survival (OS) and diseasefree survival (DFS) from prognostic factors. First, univariate analyses were performed to reveal unadjusted significant associations between prognostic variables and OS or DFS. Thereafter, variables yielding $p$ values $\leq 0.15$ in the univariate analysis were entered in the multivariate model to highlight adjusted associations between the outcome and covariates that were of borderline significance in univariate analysis. The impact of an aGvHD and cGvHD event was evaluated by including it as time-dependent covariate T_COV in the Cox model. All p values resulted from 2 -sided statistical tests, and $\mathrm{p} \leq 0.05$ was considered to be significant. OS was defined as the time between transplantation and death (independent of the cause of death). DFS was defined as CR until relapse, death or last contact, whichever occurred first. Factors analyzed in this study were age, sex, disease category (myeloid vs. lymphoid), specific underlying disease, intensity of conditioning, stem cell source, performance status, and development of GvHD.

\section{Results}

The results reported here come from 401 patients, treated at 3 different centers, who had advanced hematological malignancies [8] and who received alloHSCT. The median follow- up time was 282 days (range 3-3,607 days). After alloHSCT, $265(66.1 \%)$ patients achieved CR, 55 (13.7\%) PR, and 18 ( $4.5 \%) \mathrm{RD}$; in 1 patient the disease was stable. Of the $401 \mathrm{pa}-$ tients, 268 (66.8\%) died after alloHSCT. All 401 patients were evaluable for disease response. Of these, 147 patients $(36.7 \%)$ experienced relapse after transplantation. Kaplan-Meier estimates of OS and DFS are illustrated in fig. 1. The estimated 2- and 5-year OS were 37.1 and $27.3 \%$ (fig. 1a). The probability of DFS at 2 and 5 years was 29.1 and $21.1 \%$, respectively (fig. 1b).

Univariate analysis identified the underlying malignancy, the intensity of conditioning and development of aGvHD or cGvHD as factors with potential impact on OS. In the multivariate analysis (table 2 ), only the underlying malignancy $(p<0.001)$ and the development of aGvHD $(p<0.001)$ were confirmed as significant parameters. Patients who developed aGvHD of grade III or IV had a significantly worse OS compared to patients with aGvHD of grade 0 , I, or II. However, if aGvHD was treated as a time-dependent variable in multivariate analysis, the impact on OS was lost ( $p=0.978)$.

Diseases with the best outcome were MPS and NHL followed by AML and MM. None of the patients with advanced ALL or HD who were not in remission showed a long OS, although the number of patients with HD $(n=8)$ included in the analysis was rather small. In $70 \%$ of patients with ALL ( sample size $n=47$ ) alloHSCT was performed in the presence of active relapsed disease, which may explain the worse outcome. In addition, only $17.0 \%$ of patients with ALL developed cGvHD.

The development of cGvHD was identified as important for OS, as shown in the multivariate analysis: patients with cGvHD showed a trend towards a better OS $(p=0.085$, table $2 \mathrm{a}$ ). On the other hand, the intensity of conditioning (fig. 2a), which was identified as a parameter with borderline significance in univariate analyses $(p=0.105)$, lost its impact in multivariate analysis $(p=0.697$, table $2 a)$.

For DFS, only the underlying disease had an impact in multivariate analysis $(p=0.011$, table $2 b)$. ALL $(p=0.005$, adjusted hazard ratio (aHR) 3.00), HD ( $\mathrm{p}=0.025$, aHR 3.72), MM ( $\mathrm{p}=0.055, \mathrm{HR} 2.39)$ and AML $(\mathrm{p}=0.131$, aHR 1.65) had 
Table 2. Multivariate analysis for prognostic factors for OS and DFS

\begin{tabular}{|c|c|c|c|}
\hline & $\mathrm{p}$ value & Adjusted HR & $95 \% \mathrm{CI}$ \\
\hline \multicolumn{4}{|l|}{ a) Variable for OS } \\
\hline Diagnosis & $<0.001$ & & \\
\hline Hodgkin vs. MPS & 0.003 & 4.34 & {$[1.64 ; 11.5]$} \\
\hline MM vs. MPS & 0.135 & 2.02 & {$[0.803 ; 5.07]$} \\
\hline AML vs. MPS & 0.081 & 1.68 & {$[0.938 ; 3.00]$} \\
\hline NHL vs. MPS & 0.308 & 1.42 & {$[0.725 ; 2.77]$} \\
\hline \multicolumn{4}{|l|}{ Conditioning intensity } \\
\hline RIC vs. MAC & 0.697 & 1.10 & {$[0.676 ; 1.80]$} \\
\hline Grading & $<0.001$ & & \\
\hline 1 vs. 0 & 0.757 & 1.10 & {$[0.603 ; 2.00]$} \\
\hline 2 vs. 0 & 0.325 & 1.26 & {$[0.798 ; 1.98]$} \\
\hline 3 vs. 0 & $<0.001$ & 3.02 & {$[1.86 ; 4.92]$} \\
\hline 4 vs. 0 & $<0.001$ & 8.78 & {$[4.55 ; 16.9]$} \\
\hline \multicolumn{4}{|l|}{ aGvHD (time dependent) } \\
\hline aGvHD vs. none & 0.978 & 0.993 & {$[0.617 ; 1.599]$} \\
\hline \multicolumn{4}{|l|}{ cGvHD (time dependent) } \\
\hline \multicolumn{4}{|l|}{ b) Variable for DFS } \\
\hline Diagnosis & 0.011 & & \\
\hline Hodgkin vs. MPS & 0.025 & 3.72 & {$[1.18 ; 11.7]$} \\
\hline ALL vs. MPS & 0.005 & 3.00 & {$[1.39 ; 6.50]$} \\
\hline MM vs. MPS & 0.055 & 2.39 & {$[0.98 ; 5.80]$} \\
\hline AML vs. MPS & 0.131 & 1.65 & {$[0.862 ; 3.16]$} \\
\hline NHL vs. MPS & 0.823 & 1.09 & {$[0.506 ; 2.36]$} \\
\hline \multicolumn{4}{|l|}{ Conditioning intensity } \\
\hline RIC vs. MAC & 0.192 & 0.704 & {$[0.415 ; 1.19]$} \\
\hline \multicolumn{4}{|l|}{ aGvHD (time dependent) } \\
\hline aGvHD vs. none & 0.621 & 0.830 & {$[0.396 ; 1.74]$} \\
\hline \multicolumn{4}{|l|}{ cGvHD (time dependent) } \\
\hline Extensive or limited vs. none & 0.199 & 0.667 & {$[0.359 ; 1.24]$} \\
\hline
\end{tabular}

OS = overall survival, DFS = disease-free survival, HR = hazard ratio (HR for each risk factor is adjusted for all other factors in the multivariate model $), \mathrm{CI}=$ confidence interval, MPS = myeloproliferative syndrome, $\mathrm{ALL}=$ acute lymphoblastic leukemia, $\mathrm{MM}=$ multiple myeloma,

$\mathrm{AML}=$ acute myeloid leukemia, $\mathrm{NHL}=$ non-Hodgkin lymphoma, $\mathrm{RIC}=$ reduced-intensity conditioning, $\mathrm{MAC}=$ myeloablative conditioning, $\mathrm{aGvHD}=$ acute graft-versus-host disease, $\mathrm{cGvHD}=$ chronic GvHD.

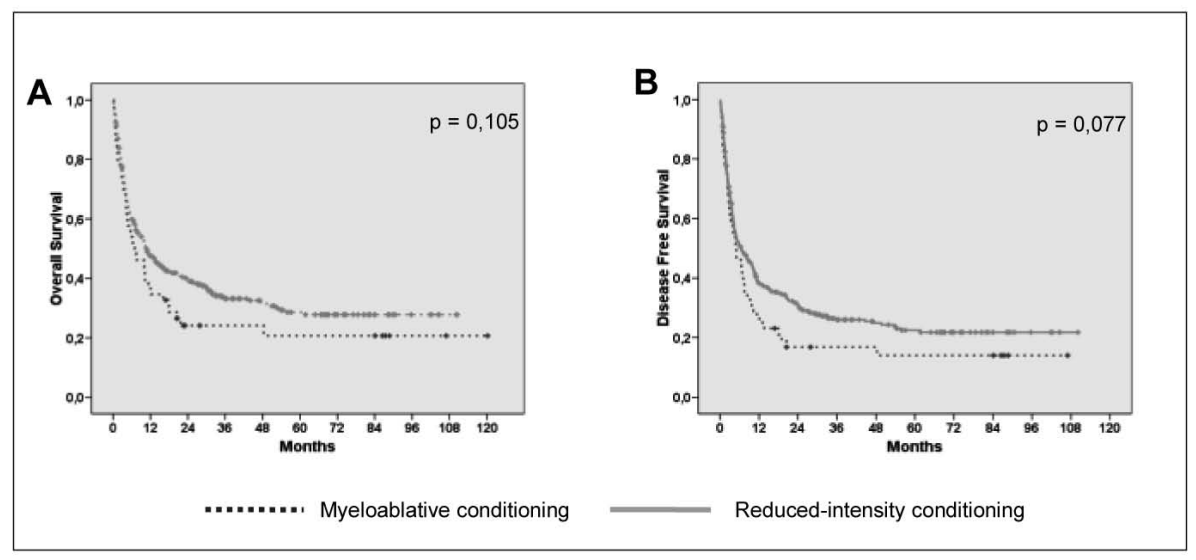

Fig 2. Univariate analysis of OS (A) and DFS (B) for patients with myeloablative conditioning (MAC) or reduced-intensity conditioning (RIC). a negative impact on OS in multivariate analysis (table 2) compared to MPS. However, development of cGvHD showed a trend for a better DFS in the multivariate analysis $(\mathrm{p}=0.199$; aHR $0.667 ; 95 \%$ confidence interval (CI) $[0.359 ; 1.24]$ ), while the intensity of the conditioning system was not a significant factor for DFS in the multivariate model $(\mathrm{p}=0.192$, aHR 0.704 ; CI $[0.415 ; 1.19])$.

None of the remaining parameters evaluated in this analysis (age, donor-recipient gender, disease category (myeloid vs. lymphoid), stem cell source or performance status) had a borderline impact on DFS or OS in univariate analysis.

\section{Discussion}

Since the outcome for patients with advanced hematological disease is poor, an alloHSCT is often performed as the only remaining curative option. However, this approach remains debatable [11] because of the severe adverse effects, the questionable outcome and the costs. Therefore, to facilitate the decision for or against an alloHSCT in a particular situation, knowledge of the general outcome in a given disease situation is crucial. The aim of this analysis was to identify parameters with prognostic influence after alloHSCT in advanced hematological diseases to identify malignancies in advanced stages in which alloHSCT is a reasonable option. 
The OS and the DFS at 2 and 5 years in this study was 37.1 and $27.3 \%$, and 29.1 and $21.1 \%$, respectively. In a study by Schmid et al. [12], 103 patients with refractory AML received a sequential regimen of chemotherapy, RIC for alloHSCT, and prophylactic donor lymphocyte transfusion. In that study, with a 25 -month median follow-up, OS at 2 years was $40 \%$ and at 4 years $32 \%$; the respective leukemia-free survival (LFS) was 37\%, and 30\%. Oyekunle et al. reported the results of 44 patients with refractory acute leukemia who underwent alloHSCT after MAC [13]. OS and progression-free survival (PFS) after 5 years was 28 and $26 \%$, respectively. Hosing et al. identified tumor burden as a prognostic factor for survival after a second transplant for AML. The OS in this group was 6 months, but a subset of patients who had a low leukemia burden at the time of the second transplant had a 5-year survival of $25 \%$ compared to $12 \%$ in those with a high leukemia burden [5]. Robinson et al. [14] reported the outcome for 188 patients with NHL after alloHSCT; the OS after 2 years was $50 \%$. The probability of having RD after 1 year for patients with chemoresistant and chemosensitive disease were 75 and $25 \%$, respectively $(\mathrm{p}=0.001)$. The PFS at 1 year was $46 \%$, and was significantly better for those with chemosensitive HD and low-grade NHL. Kennedy et al. reported the results of patients with advanced MM after alloHSCT following MAC [15]. Median OS, PFS, and event-free survival (EFS) were 28, 66 and 13 months, respectively, with a 5-year OS, PFS, and EFS of 40, 54 and $24 \%$.

Through a multivariate analysis, we identified ALL as an underlying disease and the development of aGvHD as significant factors impairing OS. cGvHD showed a trend towards a better OS and DFS (table 2). On the other hand, the intensity of conditioning appeared to have no impact on OS or DFS (fig. 2a and b, table 2). This may indicate the effectiveness of immunological effects against advanced diseases, and the susceptibility of heavily pretreated patients to treatment-related mortality induced by standard-dose MAC. The failure to achieve long-term OS in patients with advanced ALL may have been due to the low rate of cGvHD in the evaluated cohort. However, a recent publication demonstrated a complete failure of haploidentical HSCT in patients with ALL who were not in remission, indicating the limitations of a graft versus leukemia effect in patients with ALL [16]. From these results it can be assumed that for patients with advanced ALL achievement of a CR with low disease burden pre-transplant is crucial for OS [17].

The results we present here are in line with others, e.g. the reported outcome of 71 patients after a second alloHSCT [18]. The predicted OS and transplant-related mortality (TRM) at 2 years were 28 and $27 \%$, respectively. TRM was significantly lower in those who relapsed late following the first alloHSCT ( 2 years: 17 vs. $38 \%$ in early relapses; $p=0.03$ ). 2 factors were significantly associated with a better OS: late relapse $(p=0.014)$ and cGvHD following the second transplant $(p=0.014)$. Another study also showed the effect of cGvHD on the outcome after alloHSCT [19]. In a retrospec- tive analysis of the International Bone Marrow Transplant Registry, patients with extensive cGvHD experienced fewer relapses at the expense of higher treatment-related mortality.

Kahl et al. used a regimen that relied virtually entirely on graft versus tumor effects for eradicating malignancies [20]. In a retrospective analysis that included 834 patients (median age, 55; range, 5-74 years) with hematological malignancies, patients were grouped in different risk groups for relapse after non-myeloablative alloHSCT, implying a variable sensitivity for GvHD [20]. Patients with chronic lymphocytic leukemia (CLL) and MM in CR, low-grade or mantle cell NHL $(\mathrm{CR}+\mathrm{PR})$, or high-grade NHL in CR had the lowest relapse rates (0.00-0.24; low risk). In contrast, patients with advanced myeloid and lymphoid malignancies had relapse rates of $>0.52$ (high risk). Patients with lymphoproliferative diseases not in CR (except HD and high-grade NHL) and myeloid malignancies in CR had rates of $0.26-0.37$ (standard risk). Diseases grouped in the high-risk group (AML, MPS) had a better outcome in our study, probably because of the higher portion of RIC included here. It could be argued that after non-MAC, the numbers of cancer cells present at HSCT shift the balance in their favor, and they 'outproliferate' the donor cytotoxic immune cells in a majority of patients. Therefore, a higher intensity of conditioning (RIC, but probably not MAC) is required for optimal tumor cell control and for the establishment of an adequate immune response.

In conclusion, we found a significant proportion of patients with advanced hematological diseases who achieved longterm remission after alloHSCT, although the subgroup of patients with ALL did not benefit from alloHSCT. Since the development of cGvHD, but not the intensity of the conditioning, improved the outcome in this setting, it appears that chemotherapy only plays a role for immediate disease control, whereas immunological mechanisms are essential for the long-term control. Therefore, to further improve the outcome in these patients, transplant protocols with a focus on the immunological rather than cytotoxic effect are urgently needed.

\section{Acknowledgement}

The authors would like to express special thanks to Prof. R. Brand from the Department of Medical Statistics, Leiden (Netherlands) for his excellent support and detailed help to create a command syntax file for including cGvHD as a time-dependent covariate in the Cox model. E. Holler and D. Wolff receive support through a grant provided by the José Carreras Foundation.

\section{Disclosure Statement}

A. Nogai and I. Hilgendorf have received travel reimbursement to conferences from Medac GmbH. M. Freund has received a study grant and payment for lectures from Medac $\mathrm{GmbH}$. C. Junghanss has received research funding from Bayer Healthcare and payment for lectures from Novartis and BMS. All other authors declare no competing financial interests. 
1 Baron F, Storb R, Little MT: Hematopoietic cell transplantation: Five decades of progress. Arch Med Res 2003;34:528-544.

2 Bensinger WI, Martin PJ, Storer B et al.: Transplantation of bone marrow as compared with peripheral-blood cells from HLA-identical relatives in patients with hematologic cancers. N Engl J Med 2001;344:175-181.

3 Bensinger WI, Storb R: Allogeneic peripheral blood stem cell transplantation. Rev Clin Exp Hematol 2001;5:67-86.

4 Goldman JM, Gale RP, Horowitz MM et al.: Bone marrow transplantation for chronic myelogenous leukemia in chronic phase. Increased risk for relapse associated with T-cell depletion. Ann Intern Med 1988;108:806-814.

5 Hosing C, Saliba RM, Shahjahan M et al.: Disease burden may identify patients more likely to benefit from second allogeneic hematopoietic stem cell transplantation to treat relapsed acute myelogenous leukemia. Bone Marrow Transplant 2005;36: 157-162.

6 Loren AW, Bunin GR, Boudreau C et al.: Impact of donor and recipient sex and parity on outcomes of HLA-identical sibling allogeneic hematopoietic stem cell transplantation. Biol Blood Marrow Transplant 2006;12:758-769.

7 Anasetti C: What are the most important donor and recipient factors affecting the outcome of related and unrelated allogeneic transplantation? Best Pract Res Clin Haematol 2008;21:691-697.

8 Urbano-Ispizua A, Schmitz N, de Witte T et al.: Allogeneic and autologous transplantation for haematological diseases, solid tumours and immune disorders: definitions and current practice in Europe. Bone Marrow Transplant 2002;29:639646.

9 Przepiorka D, Weisdorf D, Martin P et al.: 1994 Consensus Conference on Acute GVHD Grading. Bone Marrow Transplant 1995;15:825-828.

10 Shulman HM, Sullivan KM, Weiden PL et al. Chronic graft-versus-host syndrome in man. A long-term clinicopathologic study of 20 Seattle patients. Am J Med 1980;69:204-217.

11 Alyea EP, Kim HT, Ho V et al.: Impact of conditioning regimen intensity on outcome of allogeneic hematopoietic cell transplantation for advanced acute myelogenous leukemia and myelodysplastic syndrome. Biol Blood Marrow Transplant 2006;12: 1047-1055.
12 Schmid C, Schleuning M, Schwerdtfeger R et al.: Long-term survival in refractory acute myeloid leukemia after sequential treatment with chemotherapy and reduced-intensity conditioning for allogeneic stem cell transplantation. Blood 2006; 108:1092-1099.

13 Oyekunle AA, Kroger N, Zabelina T et al.: Allogeneic stem-cell transplantation in patients with refractory acute leukemia: a long-term follow-up. Bone Marrow Transplant 2006;37:45-50.

14 Robinson SP, Goldstone AH, Mackinnon S et al.: Chemoresistant or aggressive lymphoma predicts for a poor outcome following reduced-intensity allogeneic progenitor cell transplantation: An analysis from the Lymphoma Working Party of the European Group for Blood and Bone Marrow Transplantation. Blood 2002;100:4310-4316.

15 Kennedy GA, Butler J, Morton J et al.: Myeloablative allogeneic stem cell transplantation for advanced stage multiple myeloma: Very long-term follow up of a single center experience. Clin Lab Haematol 2006;28:189-197.

16 Klingebiel T, Cornish J, Labopin M et al.: Results and factors influencing outcome after fully haploidentical hematopoietic stem cell transplantation in children with very high-risk acute lymphoblastic leukemia: Impact of center size: An analysis on behalf of the Acute Leukemia and Pediatric Disease Working Parties of the European Blood and Marrow Transplant group. Blood 2010;115:3427-3446.

17 Bader P, Kreyenberg H, Henze GHR et al.: Prognostic value of minimal residual disease quantification before allogeneic stem-cell transplantation in relapsed childhood acute lymphoblastic leukemia: The ALL-REZ BFM Study Group. J Clin Oncol 2009;27:377-384.

18 Shaw BE, Mufti GJ, Mackinnon S et al.: Outcome of second allogeneic transplants using reduced-intensity conditioning following relapse of haematological malignancy after an initial allogeneic transplant. Bone Marrow Transplant 2008;42:783-789.

19 Lee SJ, Klein JP, Barrett AJ et al.: Severity of chronic graft-versus-host disease: Association with treatment-related mortality and relapse. Blood 2002;100:406-414.

20 Kahl C, Storer BE, Sandmaier BM et al.: Relapse risk in patients with malignant diseases given allogeneic hematopoietic cell transplantation after nonmyeloablative conditioning. Blood 2007;110: 2744-2748. 